INFLAMMATION

\section{Inflammasome activation in AF}

Inflammation is a well-known independent risk factor for atrial fibrillation (AF), but the causal role of inflammation in AF initiation and maintenance is not well understood.

Now, $\mathrm{Na} \mathrm{Li}$ and colleagues show that activation of the NLRP3 inflammasome in cardiomyocytes leads to electrophysiological abnormalities associated with the pathogenesis of AF. "To our knowledge, this is the first study to demonstrate a causal link between the NLRP3 inflammasome (an innate inflammation signalling complex) and the pathophysiology of AF," says Li.

$\mathrm{Li}$ and colleagues found that the activity of the NLRP3 inflammasome was higher in atrial cardiomyocytes from patients with paroxysmal or long-lasting persistent $\mathrm{AF}$ than in cardiomyocytes from individuals in normal sinus rhythm and with no history of AF. Mice with a cardiomyocyte-specific knock-in of a constitutively active form of NLRP3 developed spontaneous premature atrial contractions and inducible $\mathrm{AF}$, indicating that cardiomyocyte-specific activation of the NLPR3 inflammasome is sufficient to promote a substrate for AF. Constitutive activation of the NLPR3 inflammasome in cardiomyocytes promoted ectopic activity, abnormal calcium release from the sarcoplasmic reticulum, shortening of the atrial effective refractory period, and atrial hypertrophy. Treatment with an NLRP3 inflammasome-specific inhibitor attenuated $\mathrm{AF}$ development in these mice. Finally, cardiomyocyte-specific Nlrp3 knockdown prevented AF development in this mouse model and in a well-characterized mouse model of spontaneous AF.

"Our study establishes a link between the altered inflammasome activity in cardiomyocytes and the well-known electrophysiological changes and calcium mishandling associated with the pathogenesis of AF," concludes $\mathrm{Li}$. The research team plans to investigate the factors that lead to the activation of the cardiomyocyte NLRP3 inflammasome in $\mathrm{AF}$ and to evaluate this pathway as a potential therapeutic target for this condition.

Irene Fernández-Ruiz

ORIGINAL ARTICLE Yao, C. et al. Enhanced cardiomyocyte NLRP3 inflammasome signaling promotes atrial fibrillation. Circulation https://doi.org/10.1161/CIRCULATIONAHA. 118.035202 (2018) FURTHER READING Hu, Y. F. et al. Inflammation and the pathogenesis of atrial fibrillation. Nat. Rev. Cardiol. 12 230-243 (2015)

\title{
Enduring FAME of FFR-guided PCI
}

Patients with stable coronary artery disease (CAD) and a fractional flow reserve (FFR) $\leq 0.80$ benefit from revascularization plus medical therapy, whereas those with an FFR $>0.80$ have a favourable outcome with medical therapy only. These 5 -year outcomes of the FAME 2 trial were presented at the EuroPCR conference in Paris, France, and simultaneously published in NEJM.

A total of 888 patients with an FFR $\leq 0.80$ were randomly assigned to percutaneous coronary intervention (PCI) plus medical therapy or to medical therapy only.

Patients with an FFR $>0.80$ received medical therapy only and were entered into a registry. After 5 years of follow-up, the rate of the primary end point (a composite of death, myocardial infarction, or urgent revascularization) was lower in the PCI group than in the medical-therapy group $(13.9 \%$ versus $27.0 \%$; HR 0.46, 95\% CI 0.34-0.64). This difference was driven by a lower rate of urgent revascularization in the PCI group $(6.3 \%$ versus $21.1 \%$; HR $0.27,95 \% \mathrm{CI}$ $0.18-0.41)$. Of note, PCI was also associated with a lower rate of spontaneous myocardial infarction $(6.5 \%$ versus $10.2 \%$; HR 0.62 ,
$95 \%$ CI 0.39-0.99). By contrast, mortality was similar in the two groups (5.1\% versus $5.2 \%)$. The rate of the primary end point was not significantly different in the group undergoing PCI compared with the registry cohort (13.9\% versus $15.7 \%$ ).

"In patients with stable CAD, an initial FFR-guided PCI strategy resulted in a sustained clinical benefit," conclude the investigators. "Our results contradict the general belief that abrupt coronary occlusions occur predominantly at sites of mild stenosis and hence that the treatment of severe lesions may not prevent myocardial infarction." These 5-year data conclude the follow-up of the FAME 2 trial. Recruitment into the FAME 3 trial, designed to compare CABG surgery and FFR-guided PCI in patients with three-vessel CAD, is almost complete.

Gregory B. Lim

ORIGINAL ARTICLE Xaplanteris, P. et al. Five-year outcomes with $\mathrm{PCl}$ guided by fractional flow reserve. N. Engl. J. Med. https://doi.org/10.1056/NEJMoa1803538 (2018) FURTHER READING Katritsis, D. G. et al. Revascularization in stable coronary disease: evidence and uncertainties. Nat. Rev. Cardiol. https://doi.org/10.1038/s41569-018-0006-z (2018)

\section{INTERVENTIONAL CARDIOLOGY}

\section{Drug-eluting stents versus bare-metal stents for vein-graft $\mathrm{PCI}$}

Saphenous vein grafts (SVGs) are commonly used for $C A B G$ surgery, but they are prone to occlusion and show high rates of failure. Strategies to treat SVG stenosis include percutaneous coronary intervention $(\mathrm{PCl})$ with stent implantation. A new study reveals that patients who receive drug-eluting stents (DES) for $\mathrm{PCl}$ of SVG lesions have similar clinical outcomes to patients who receive bare-metal stents (BMS).

Previous efforts to compare the performance of the two types of stents resulted in conflicting results owing to study limitations. "There was equipoise as to whether DES would provide better outcomes than BMS in SVGs," explains Emmanouil Brilakis, corresponding author of the study. The double-blind, randomized trial included 597 patients, with at least one de-novo, clinically significant SVG lesion, who were randomly assigned to receive DES or BMS for $\mathrm{PCl}$. After 12 months, no significant difference was found between the two groups for the primary combined end point of cardiac death, target-vessel myocardial infarction, or target-vessel revascularization, with an incidence of $17 \%$ in the DES group and $19 \%$ in the BMS group ( $\mathrm{HR} 0.92,95 \% \mathrm{Cl} 0.63-1.34$, $P=0.70$ ). The risks of stent thrombosis and stroke were also similar between the two groups.

"We were surprised by those findings, as we were anticipating that DES would be superior to BMS as has been shown for multiple other coronary lesion types," says Brilakis. The specific pathophysiology of atherosclerosis in a graft contributes to the lesion-specific efficiency of DES for $\mathrm{PCl}$ and supports the use of alternative options to treat graft failure. "Given the overall poor outcomes after SVG stenting, treating the native coronary arteries supplying the same myocardial territory instead may be advantageous," concludes Brilakis.

\section{Alexandra Le Bras}

ORIGINAL ARTICLE Brilakis, E. S. et al. Drug-eluting stents versus bare-metal stents in saphenous vein grafts: a doubleblind, randomised trial. Lancet 391, 1997-2007 (2018) 